\title{
Performative Erfahrungsfelder im Primar- und Sekundarschulwesen
}

\section{Micha Fleiner}

Elena, Denise (2012): Dramapädagogik für Englisch in der Grundschule. Das Klassenzimmer als Bühne. Mit Rollenspielen kommunikative Kompetenz fördern. Buxtehude: AOL. ISBN: 978-3-403-10029-4

Elena, Denise (2011): Dramapädagogik für Englisch in der Sekundarstufe. Das Klassenzimmer als Bühne. Mit Rollenspielen kommunikative Kompetenz fördern. Buxtehude: AOL. ISBN: 978-3-403-10028-7

Unterzieht man die fachpraktische Lehrliteratur der vergangenen Jahre unter Einschluss der Fülle von begleitenden Arbeits-, Übungs- und Unterrichtsmaterialien - einer kritischen Sichtung, kann man sich des Eindrucks nicht erwehren, dass der Einsatz dramapädagogischer Lehr- und Lernangebote in der deutschsprachigen Schullandschaft in zunehmendem Maße an Bedeutung gewinnt. Für theateraffine Fremdsprachenlehrerinnen und -lehrer mit der Zielsprache Englisch liegen nun zwei weitere Werke - Dramapädagogik für Englisch in der Grundschule sowie Dramapädagogik für Englisch in der Sekundarstufe bereit, die Interesse für das szenische Interaktionspotenzial im fremdsprachlichen Klassenraum zu wecken beabsichtigen. ${ }^{1}$ Das Kernanliegen der Autorin - Denise Elena ist praktizierende Fremdsprachenlehrkraft und diplomierte Theaterpädagogin zugleich - besteht in der Vermittlung eines grundlegenden Zuganges zu dramapädagogischem Fremdsprachenunterricht: Lehrpersonen mit Interesse an performativen Erfahrungsräumen soll Gelegenheit geboten werden, dramapädagogische Basiskompetenzen zu erwerben und ausgewählte Inszenierungstechniken situationsadäquat im eigenen Englischunterricht anzuwenden. Dieser Grundgedanke spiegelt sich denn auch im inhaltlichen Aufbau beider Ausgaben wider, der von Anbeginn einer konsequenten Zweigliedrigkeit folgt: So bildet der theoriegestützte Abschnitt (Kapitel 2-4) gleichsam einen Ausgangs- und Anknüpfungspunkt für den anwendungsbezogenen Hauptteil (Kapitel 5) des Werkes, in dessen Verlauf der Leser Einblicke in acht methodisch vorstrukturierte Theaterworkshops gewinnt.

Dem Vorwort (Kapitel 1) folgen einzelne Überlegungen $\mathrm{zu}$ bestehenden Curricula und allgemeinen Rahmenvorgaben im Bereich des schulbezogenen Sprachunterrichts (Kapitel 2), die ausnahmslos introduktiven Charakter tragen.

\footnotetext{
${ }^{1}$ Der wesentliche Unterschied zwischen beiden Werke liegt in erster Linie in der schulstufengerechten Auswahl und Aufbereitung der Workshop-Themen. Beide Ausgaben folgen indes einer identischen Grundstruktur und behandeln im theoretischen Part - geringfügiger Abwandlungen ungeachtet - deckungsgleiche Inhalte.
} 
Hieran schließt sich ein nicht ganz unproblematischer Definitionsansatz des Kernbegriffes Dramapädagogik (Kapitel 3) an, der auf knappem Raum in Abgrenzung zum Inhalts- und Aufgabenbereich der Theaterpädagogik dargestellt wird; eine begriffliche Opposition, die - wohlgemerkt, auch zum gegenwärtigen Zeitpunkt - nicht selten Gegenstand lebhaft geführter Fachdiskussionen bildet. Im weiteren Verlauf des dritten Kapitels skizziert die Verfasserin Bezugslinien zwischen aktuellen Kompetenzfeldern des schulischen Fremdsprachenunterrichts und dem Begründungs- beziehungsweise Einsatzpotenzial dramapädagogischer Formen des Lehrens und Lernens. Eine nutzbringende Argumentationsgrundlage gerade auch für all jene Lehrpersonen, die sich ob ihres dramapädagogisch akzentuierten Fachunterrichts einem - sei es vonseiten der Schulleitung, des Kollegiums oder der Elternschaft - erhöhten Legitimationsdruck ausgesetzt sehen.

Die Frage nach einem fruchtbaren Implementierungsprozess ästhetischer Handlungsfelder in der konkreten Unterrichtssituation wird sodann im abschließenden Theoriepart (Kapitel 4) aufgegriffen: Anhand einer Tour d'Horizon durch das dramapädagogische Basisvokabular (z. B. Raum, Form, Akteure, Kontext, Fiktion, Methodenansätze etc.) werden Anforderungen, Inhalte und Ziele des darstellungsbezogenen Fremdsprachenunterrichts nachgezeichnet, d. h. der unterrichtsrelevante Handlungsrahmen für die Durchführung der themenspezifischen Workshops gesetzt. Obschon die Autorin an anderer Stelle die Notwendigkeit eines sanktionsfreien Gestaltungsfeldes unterstreicht, wäre es in diesem Kontext gewiss aufschlussreich gewesen, abwägende Betrachtungen zur Leistungsdiagnose, d. h. zum Verhältnis von performativ-ästhetischen Arbeitsformen und schulischen Bewertungsstrukturen, einzubeziehen. ${ }^{2}$

Im ausführlichsten Teil des Buches (Kapitel 5) präsentiert Elena eine achtteilige Serie von flexibel kombinierbaren Praxisbausteinen, denen allgemeine Hinweise in Bezug auf das Arbeits- und Kommunikationsverhalten innerhalb der Lerngruppe sowie auf den adäquaten Umgang mit Präsentationen, Arbeitskritik und sprachlichen Ungenauigkeiten vorangestellt werden. Die Beschreibungen der einzelnen Praxiseinheiten - stets einem dreiphasigen Gliederungsprinzip (Einstieg, Hauptphase, Abschluss) folgend - enthalten Informationen zum thematischen Kontext, zur zeitlichen Planung sowie zu begleitenden Materialien. Begrüßenswert hierbei ist die thematisch umfangreiche Bandbreite der Workshops, die sowohl improvisatorische (z. B. Entwicklung spontaner Sprechfertigkeiten) als auch sprachkorrektheitsbezogene (z. B. Bildung von Zeitformen, Gebrauch von Modalverben) und wortschatzgestützte (z. B. Erschließung von Wortgruppen und Bedeutungsfeldern) Zieldimensionen umfasst. Gleichwohl muss hervorgehoben werden, dass der inhaltliche Komplexitätsgrad und damit die Erwartungen an das dramapädagogische Kompetenzprofil d er $\mathrm{L}$ ehrkraft $\mathrm{m}$ itunter $\mathrm{b}$ eträchtlich $\mathrm{v}$ ariieren: $\mathrm{F}$ ür einen erstmaligen Einsatz szenisch-dramatischer Arbeitsformen im eigenen Englischunterricht erweisen sich einzelne der aufgeführten Workshop-Elemente daher

\footnotetext{
${ }^{2}$ Zum generellen Verhältnis von szenisch-dramatischen Lernformen und Möglichkeiten der Leistungsdiagnose vgl. etwa Fleming (2011: 140 ff.).
} 
nur in beschränktem Umfang als geeignet.

Fazit: Der übergreifende Zielhorizont der beiden hier erörterten Werke, theaterinteressierten Fremdsprachenlehrenden einen anwendungsnahen Zugang zu performativen Gestaltungsmöglichkeiten im Primar- und Sekundarschulwesen zu ermöglichen, wird erfüllt. Sicherlich hätte die interessierte Leserschaft im dritten und vierten Kapitel streckenweise einen weniger eng gesteckten theoretischen Bezugsrahmen dankend zur Kenntnis genommen. Und sicherlich muss sich jede Lehrkraft, die Interesse an dramapädagogischem Fremdsprachenunterricht hegt, in grundsätzlicher Hinsicht die Frage stellen, ob ein workshopzentrierter Vermittlungsansatz der persönlichen Lehrmethodik gerecht $\mathrm{zu}$ werden vermag. Doch seien die Vorzüge der beiden vorliegenden Veröffentlichungen abschließend noch einmal in verdichteter Form wiedergegeben: Klare Strukturierung und leserfreundliche Gestaltung der Inhalte, abwechslungsreiche Auswahl der didaktischen Materialien unter Berücksichtigung der Lehrer- und Schülerperspektive sowie ein reiches Maß an Praxisorientierung für eine zeitnahe Umsetzung der dramapädagogischen Impulse im englischsprachigen Klassenzimmer. Man darf gespannt sein, zu welchem Zeitpunkt ein der französischen, spanischen oder italienischen Zielsprache verpflichtetes Arbeitsbuch vergleichbaren Formates auf dem hiesigen Lehrbuchmarkt Eingang finden wird.

\section{Bibliografie}

Fleming, Mike (2011): Starting Drama Teaching. 3. Aufl. London \& New York: Routledge. 